\title{
BIODEGRADABLE FOOD PACKAGING MEMBRANE FROM BACTERIAL CELLULOSE AND BACTERIOCIN FOR PRESERVING RAW FOOD
}

\author{
Nhu Thi Huynh Nguyen, Nhu Quynh Nguyen, Nhi Thi Yen Nguyen, \\ Phuong Hoang Ngoc Nguyen
}

Faculty of Biology and Biotechnology, University of Science, VNU-HCM

Received in: 30/6/2019; Revised on: 11/8/2019;

Accepted on: 26/8/2019)

\section{Summary}

Raw foods such as fish and meat sold at local markets are easily pathogenically infected because retailers do not keep them in proper food preservation equipment. In addition, food packaging using single-use plastic grocery bags is a burden for waste management and aggravates the "white pollution". In this research, we created biodegradable bacterial cellulose membrane (BCm) loaded with raw bacteriocins that were collected from isolated lactic acid bacteria (LAB) and test their ability to preserveraw fish and meat.

The $2 \mathrm{~mm}$ thick and $94 \%$ wet BC membrane was obtained from the surface fermentation of Acetobacterxylinum in the medium containing $50 \%$ of coconut water and $15 \%$ sucrose after 03 days. The isolated LAB from sour cabbage, kimchi and yogurt were microscopically observed, biochemically characterized and species identified by mass spectrometry. The raw bacteriocin from Lactobacillus plantarum showing the most antimicrobial capability among the isolates could inhibit the growth of 3 tested strains of Escherichia coli, Staphylococcus aureus and Bacillus subtilis at the antimicrobial activity of $100 \mathrm{AU} / \mathrm{ml}$ by agar-well diffusion method. BCm treated with the bacteriocins of $100 \mathrm{AU} / \mathrm{ml}$ in 45 minutes was proved to be able to well preserve fish for 24 hours and pork for 18 hours at ambient temperatures, following Vietnam Standard for catfish fillet (TCVN 8338:2010) and for meat (TCVN 7046:2009).

Keywords: Bacteriocin, bacterial cellulose, biodegradation, food preservation.

\section{INTRODUCTION}

According to the Vietnam Ministry of Health, approximately 200 food poisoning outbreaks with 30 deaths happened annually from 2011 to 2014 (JAHR, 2015). Among chemical preservatives for foods, bacteriocins from LAB are economic, affordable and safe for health. Bacteriocins are peptides or proteins that can inhibit other microorganisms (Cotter et al., 2005). Their mechanisms can be divided into bactericidal effect of cell lysis or non-cell lysis e.g. bacteriostatic (da Silva Sabo et al., 2014). Bacterial cellulose synthesized by Acetobacterxylinum can be used for replacing plastic grocery bags (Azeredo et al., 2019). BCm loaded with nisin, a commercial pure bacteriocin was used to test against Staphylococcus aureus, Escherichia coli and Pseudomonas aeruginosaby agar diffusion assay and antioxidant activity (dos Santos et al., 2018). Bacterial cellulose loaded with bacteriocin from Lactococcus lactic could preserve under-cooked meat (Huong Thuy Nguyen et al, 2018). In this research, raw bacteriocin was loaded in BC to replace plastic grocery bags and preserve raw foods including catfish and pork.

${ }^{1}$ Tel: 0708829662 Email:nhnphuong@hcmus.edu.vn 


\section{MATERIALS AND METHODS}

\subsection{Isolation of lactic acid bacteria and bacteriocin production}

Colonies of LAB from at least 10 different sources of pickled cabbage, kimchi and yogurt from local markets were screened on $\mathrm{MRS}-\mathrm{CaCO}_{3}$ agar. Then, they were observed morphologically under microscopy and performed biochemical tests for LAB following Vietnam Standard (TCVN 9633:2013). Isolated clones were identified by matrix-assisted laser desorption/ionization mass spectrometry (MALDI-TOF MS) at Oxford University Clinical Research Unit Vietnam (OUCRU-VN).

\subsection{Assessment of antimicrobial activity from isolated LAB by minimum inhibitory concen- tration and well diffusion agar}

The antimicrobial activities against indicator strains including E. coli, B. subtilis and S. aureus were assessed by minimal inhibitory concentration and agar-well diffusion method (Tagg and McGiven, 1971). Isolated LABs were cultured in MRS broth and incubated at ambient temperature for $72 \mathrm{~h}$. Supernatants were collected by centrifuging at $40^{\circ} \mathrm{C}$, adjusted to $\mathrm{pH}$ 6.5-7 and semi-purified by $0.45 \mu \mathrm{m}$ pore size filter membranes. Each indicator strain was spread on nutrient agar plate; then $80 \mu 1$ of the raw bacteriocin was added into agar wells ( $5 \mathrm{~mm}$ diameter) and incubated at ambient temperature for 24 hours. The antimicrobial effects of bacteriocin were assessed via the inhibition zones $(\mathrm{mm})$ around the antimicrobial wells onthe agar plates. Antimicrobial activity was determined by the serial 2-fold dilution method: the highest concentration in a range of diluted continuous solutions at which the indicator microorganism is still inhibited in a volume unit (AU/ml) (Vignolo, 1995).

\subsection{Making and treating $\mathrm{BC}$ for preserving catfish and pork}

$1 \mathrm{~L}$ medium for statically culturing Acetobacterxylinum contained $2 \mathrm{~g}$ yeast extract, $8 \mathrm{~g}$ $\left(\mathrm{NH}_{4}\right)_{2} \mathrm{SO}_{4}, 2 \mathrm{~g}\left(\mathrm{NH}_{4}\right)_{2} \mathrm{HPO}_{4}, 500 \mathrm{ml}$ of coconut milk, $5 \mathrm{ml}$ of acetic acid and different percentages of sucrose to investigate the suitable thickness of $\mathrm{BCm}$. After $\mathrm{BCm}$ wash harvested, it was drained, autoclaved and loaded with bacteriocin.

Catfish and pork were purchased at the local market (Thu Duc, Ho Chi Minh City) and prepared with $500 \mathrm{~g}$ each sample. Each sample was preserved with BCm-bacteriocin from thei solated LAB producing highest antimicrobial bacteriocin at ambient temperature. Samples were tested for aerobic plate count (APC) every 6 hours for 24 hours following Vietnam Standard for catfish fillet (TCVN 8338:2010) and meat (TCVN 7046:2009).

\section{RESULTS AND DISCUSSION}

\subsection{Characterizing isolated $L A B$ and bacteriocin production}

Thei solates were picked up based on the size of $\mathrm{CaCO}_{3}$ lysis zone on agar plate and physical characteristics of specific LAB colonies such as milky white, smooth and convex. By microscopic observation and biochemical tests, the isolates were characterized following Bergey's Manual of Determinative Bacteriology before identified by MALDI-TOF MS (Table 1).

Table 1. Morphological and biochemical characteristics of the isolates

\begin{tabular}{|c|c|c|c|c|c|c|c|c|c|}
\hline Sources & \multicolumn{3}{|c|}{ Sour cabbage } & \multicolumn{3}{c|}{ Kimchi } & \multicolumn{3}{c|}{ Yogurt } \\
\hline Isolated labels & NC1 & NC2 & NC3 & NK1 & NK2 & NK3 & NY1 & NY2 & NY3 \\
\hline Gram & $(+)$ & $(+)$ & $(+)$ & $(+)$ & $(+)$ & $(+)$ & $(+)$ & $(+)$ & $(+)$ \\
\hline Cell form & Short rod & Short rod & Short rod & Short rod & Rod & Short rod & Rod & Rod & Rod \\
\hline Arrangement & $\begin{array}{c}\text { Single, } \\
\text { chain }\end{array}$ & $\begin{array}{c}\text { Single, } \\
\text { chain }\end{array}$ & $\begin{array}{c}\text { Single, } \\
\text { pair }\end{array}$ & $\begin{array}{c}\text { Single, } \\
\text { pair }\end{array}$ & $\begin{array}{c}\text { Single, } \\
\text { pair }\end{array}$ & Single, pair & $\begin{array}{c}\text { Single, } \\
\text { pair, chain }\end{array}$ & $\begin{array}{c}\text { Single, pair } \\
\text { Single, pair, } \\
\text { chain }\end{array}$ \\
\hline Lactic acid & + & + & + & + & + & + & + & + & + \\
\hline
\end{tabular}


NGHIÊN CÚUU KHOA HỌC

\begin{tabular}{|c|c|c|c|c|c|c|c|c|c|}
\hline Sources & \multicolumn{3}{|c|}{ Sour cabbage } & \multicolumn{3}{|c|}{ Kimchi } & \multicolumn{3}{|c|}{ Yogurt } \\
\hline Spore & - & - & - & - & - & - & - & - & - \\
\hline Moveable & - & - & - & - & - & - & - & - & - \\
\hline Catalase & - & - & - & - & - & - & - & - & - \\
\hline Oxidase & - & - & - & - & - & - & - & - & - \\
\hline $\begin{array}{l}\text { Nitrate } \\
\text { reductase }\end{array}$ & - & - & - & - & - & - & - & - & - \\
\hline Indole & - & - & - & - & - & - & - & - & - \\
\hline $\begin{array}{c}\text { Identification } \\
(*)\end{array}$ & $\begin{array}{c}L . \\
\text { plantarum }\end{array}$ & $\begin{array}{c}L . \\
\text { plantarum }\end{array}$ & $\begin{array}{c}L . \\
\text { plantarum }\end{array}$ & $\begin{array}{c}L . \\
\text { plantarum }\end{array}$ & $\begin{array}{c}L . \\
\text { plantarum }\end{array}$ & $\begin{array}{c}L . \\
\text { plantarum }\end{array}$ & $\begin{array}{c}L . \\
\text { paracasei }\end{array}$ & $\begin{array}{c}L . \\
\text { paracasei }\end{array}$ & $\begin{array}{c}L . \\
\text { paracasei }\end{array}$ \\
\hline
\end{tabular}

(*) Identified by MALDI-TOFMS

3.2. Screening of LAB isolates for antibacterial activity using well diffusion agar method and determining the activity units $(\mathrm{AU} / \mathrm{ml})$

All of the isolates produced bacteriocin and inhibited all three of the indicator strains of E. coli, B. subtilis, S. aureus (Table 2). In general, the isolated LABs from pickled cabbage demonstrated antimicrobial capability higher than those from kimchi and yogurt. NC2 was identified as Lactobacillus plantarum produced the highest antimicrobial activity. The antibacterial activity of $100 \mathrm{AU} / \mathrm{ml}$ from $\mathrm{NC} 2$ was assessed by the serial 2-fold dilution method on diffusion agar plate containing E. coli.

Table 2. The inhibition zones ( $\mathrm{mm}$ ) of the LAB isolates against the indicator microorganisms

\begin{tabular}{ccccc}
\hline Sources & Labels & \multicolumn{3}{c}{ Diameters of inhibition zones $\boldsymbol{\Delta D}=\boldsymbol{D}$-d $(\mathbf{m m})$} \\
\cline { 2 - 5 } & & E. coli & B. subtilis & S. aureus \\
\hline \multirow{3}{*}{ Sour cabbage } & NC1 & $6.5 \pm 1.14$ & $8.5 \pm 1.35$ & $6.3 \pm 1.51$ \\
\cline { 2 - 5 } & NC2 & $8.5 \pm 0.70$ & $8.9 \pm 0.46$ & $7.0 \pm 0.64$ \\
\cline { 2 - 5 } & NC3 & $8.3 \pm 0.95$ & $8.5 \pm 0.91$ & $6.1 \pm 0.49$ \\
\hline \multirow{3}{*}{ Yogurt } & NK1 & $6,4 \pm 0,19$ & $6.1 \pm 1.03$ & $4.6 \pm 0.86$ \\
\cline { 2 - 5 } & NK2 & $5.9 \pm 0.73$ & $6.3 \pm 0.83$ & $5.6 \pm 0.86$ \\
\cline { 2 - 5 } & NK3 & $6.2 \pm 2.61$ & $6.7 \pm 2.66$ & $4.9 \pm 1.12$ \\
\hline & NY2 & $8.5 \pm 1.41$ & $7.3 \pm 1.77$ & $6.7 \pm 2.43$ \\
\cline { 2 - 5 } & NY3 & $8.4 \pm 1.32$ & $6.2 \pm 2.22$ & $4.2 \pm 2.70$ \\
\hline
\end{tabular}

D: Diameters of inhibition zones, $d$ : Diameters of colonies

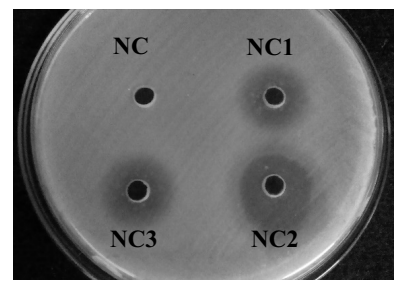

A

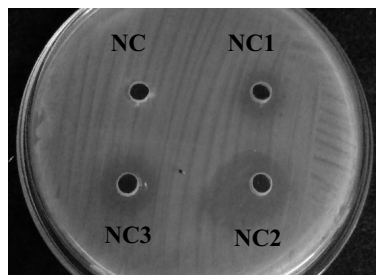

B

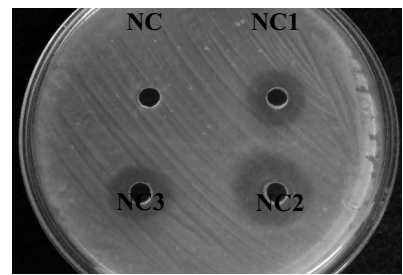

$\mathrm{C}$

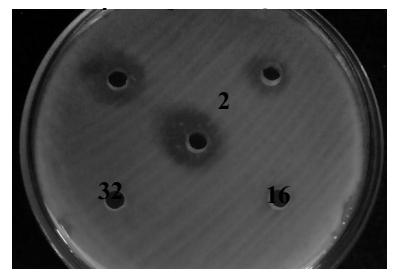

$\mathrm{D}$

Figure 1. The isolated LAB against indicator microorganism and antibacterial activity of NC2 
$N C$ : negative control; $N C 1, N C 2, N C 3$ : bacteriocin of the respective isolates; $A, B, C$ : indicators B. subtilis, S. aureus, E. coli; D: antibacterial activity of NC2 by the serial 2-fold dilution method

\subsection{Making and treating $\mathrm{BC}$ for preserving catfish and pork}

The effect of sucrose concentration and fermented time to $\mathrm{BCm}$ quality is shown in Table 3 . The $\mathrm{BCm}$ should be harvested after 3 days for fermentation with medium containing $15 \%$ sucrose. The $\mathrm{BCm}$ got 1-2 mm thick, and their area depended on fermented containers.

Table 3. The effects of time and concentration of sugar on quality $B C\left(6.3 \times 10^{3} \mathrm{~mm}^{2}\right.$ area $)$

\begin{tabular}{|c|c|c|c|c|}
\hline $\begin{array}{c}\text { Fermented } \\
\text { time } \\
\text { (day) }\end{array}$ & $\begin{array}{c}\text { Sucrose } \\
\text { concentration } \\
(\%)\end{array}$ & Characteristics & Weight (g) & $\begin{array}{c}\text { Mean } \\
\text { humidity (\%) }\end{array}$ \\
\hline \multirow{3}{*}{1} & 20 & & & \multirow{3}{*}{-} \\
\hline & 15 & Not formed & - & \\
\hline & 10 & & & \\
\hline \multirow{3}{*}{2} & 20 & Thin, not tough & - & - \\
\hline & 15 & \multirow[b]{2}{*}{ Not completed } & \multirow[b]{2}{*}{-} & \multirow[b]{2}{*}{ - } \\
\hline & 10 & & & \\
\hline \multirow{3}{*}{3} & 20 & \multirow{2}{*}{ Completed, tough, $1-2 \mathrm{~mm}$ thick } & $8.7 \pm 0.55$ & $94.8 \pm 0.15$ \\
\hline & 15 & & $7.2 \pm 0.28$ & $93.8 \pm 0.13$ \\
\hline & 10 & Thin, $<1 \mathrm{~mm}$ thick & $5.3 \pm 1.24$ & $93.0 \pm 1.68$ \\
\hline \multirow{3}{*}{4} & 20 & Completed, tough, $>2 \mathrm{~mm}$ thick & $9.7 \pm 0.83$ & $93.9 \pm 0.27$ \\
\hline & 15 & \multirow{2}{*}{ Completed, tough, $1-2 \mathrm{~mm}$ thick } & $8.0 \pm 0.25$ & $93.5 \pm 0.37$ \\
\hline & 10 & & $7.8 \pm 0.62$ & $94.4 \pm 0.70$ \\
\hline
\end{tabular}

BC loaded 100AU/ml raw bacteriocin from isolated L. plantarum (labeled as samples) for 45 minutes was used to preserve raw catfish and pork at ambient temperature. The quality of raw catfish and pork during the storage time is shown in Table 4. Both aerobic plate count (APC) in dexin catfish and pork were much lower than those in $\mathrm{C}_{1}$ (the control: $\mathrm{BCm}$ without loaded bacteriocin).

Organoleptic and microbial standards were assessed following to Vietnam Standard for catfish (TCVN 8338:2010). After 6 hours of storage, $\mathrm{C}_{1}\left(5.6 \times 10^{5} \mathrm{CFU} / \mathrm{g}\right)$ the raw catfish got rancid and mucous on surface. Meanwhile, sample $\mathrm{S}$ still met standard of organoleptic quality of catfish products and APC $\left(1.0 \times 10^{6} \mathrm{CFU} / \mathrm{g}\right)$ after 24 hours.

Like catfish, the pork qualities were assessed following TCVN 7046:2009. After 6 hours of storage, $\mathrm{C} 1$ did not meet the the permitted standard of APC and organoleptic qualities. The color of raw pork got darker, rancid and mucous on surface. Meanwhile, S still met organoleptic and APC quality $\left(1 \times 10^{6} \mathrm{CFU} / \mathrm{g}\right)$ after 18 hours. After 24-hour storage, $\mathrm{S}$ exceeded the permitted APC standard.

In summary, $\mathrm{BCm}$ loaded with the raw bacteriocins of $100 \mathrm{AU} / \mathrm{ml}$ from the isolated L. plantarumin 45 minutes could preserve raw catfish and pork for 24 hours and 18 hours respectively following Vietnam Standard for catfish fillet (TCVN 8338:2010) and meat (TCVN 7046:2009). 
Table 4. Organoleptic and microbial quality of catfish and meat

\begin{tabular}{|c|c|c|c|c|c|c|}
\hline \multirow{2}{*}{ Sample } & \multirow{2}{*}{$\begin{array}{c}\text { Storage time } \\
\text { (hour) }\end{array}$} & \multirow{2}{*}{ Label } & \multicolumn{3}{|c|}{ Organoleptic characteristics } & \multirow{2}{*}{$\begin{array}{c}A P C \\
C F U / g\end{array}$} \\
\hline & & & Color & Odor & Surface & \\
\hline \multirow{9}{*}{ CATFISH } & 0 & $\mathrm{C}_{0}$ & & Typical & & $1.1 \times 10^{5}$ \\
\hline & \multirow{2}{*}{6} & $\mathrm{C}_{1}$ & Pinkish & Less rancid odor & Less mucous & $5.6 \times 10^{5}$ \\
\hline & & $\mathrm{S}$ & & Typical & & $1.5 \times 10^{5}$ \\
\hline & \multirow{2}{*}{12} & $\mathrm{C}_{1}$ & Rosy & Less rancid odor & Less mucous & $2.6 \times 10^{6}$ \\
\hline & & $\mathrm{S}$ & & Typical & & $3.5 \times 10^{5}$ \\
\hline & \multirow{2}{*}{18} & $\mathrm{C}_{1}$ & Rosy & Rancid odor & Mucous & $3.0 \times 10^{6}$ \\
\hline & & $\mathrm{S}$ & & Typical & & $6.6 \times 10^{5}$ \\
\hline & \multirow{2}{*}{24} & $\mathrm{C}_{1}$ & Rosy & Rancid odor & Mucous & $6.5 \times 10^{6}$ \\
\hline & & $\mathrm{S}$ & & Typical & & $1.0 \times 10^{6}$ \\
\hline \multirow{9}{*}{ MEAT } & 0 & $\mathrm{C}_{0}$ & & Typical & & $6.9 \times 10^{4}$ \\
\hline & \multirow{2}{*}{6} & $\mathrm{C}_{1}$ & Pinkish & Less rancid odor & Less mucous & $1.8 \times 10^{6}$ \\
\hline & & $\mathrm{S}$ & & Typical & & $1.2 \times 10^{5}$ \\
\hline & \multirow{2}{*}{12} & $\mathrm{C}_{1}$ & Rosy & Less rancid odor & Mucous & $2.3 \times 10^{6}$ \\
\hline & & $\mathrm{S}$ & & Typical & & $5.0 \times 10^{5}$ \\
\hline & \multirow{2}{*}{18} & $\mathrm{C}_{1}$ & Rosy & Rancid odor & Mucous & $4.5 \times 10^{6}$ \\
\hline & & $\mathrm{S}$ & & Typical & & $1.0 \times 10^{6}$ \\
\hline & \multirow{2}{*}{24} & $\mathrm{C}_{1}$ & Rosy & Rancid odor & Mucous & $9.2 \times 10^{6}$ \\
\hline & & $\mathrm{S}$ & & Typical & & $1.4 \times 10^{6}$ \\
\hline
\end{tabular}

$C_{0}$ : control at 0 hour; $C_{1}$ : control according to preservation time at ambient temperature $S: B C$ membrane treated with the bacteriocins of $100 \mathrm{AU} / \mathrm{ml}$ in 45 minutes for preservation

\section{CONCLUSIONS}

Food safety risk management in Vietnam is a challenge since most of food suppliers are local retailers who have not recognized and practiced adequately the instructions of food preservation for raw foods. The research indicated $\mathrm{BC}$ membrane loaded raw bacteriocin can be an alternative material for packing of raw foods in small business and retailers to reduce single plastic bags and enhance food preservation capability. The research also provided two of isolated LABs identified as L. plantarum and L. paracasei whose bacteriocin inhibited 3 of indicator strains E. coli, S. aureus, B. subtilis. Raw bacteriocin from L. plantarum showed the most antimicrobial capability among the isolates with the antibacterial activity of $100 \mathrm{AU} / \mathrm{ml}$ on E. coli. The suitable medium containing $50 \%$ of coconut milk and $15 \%$ sucrose for producing $\mathrm{BC}$ membrane by the static fermentation of Acetobacterxylinum was also assessed. $\mathrm{BC}$ membrane loaded with the raw bacteriocin of $100 \mathrm{AU} / \mathrm{ml}$ could preserve raw catfish and 
pork for 24 hours and 18 hours respectively following Vietnam Standard for catfish fillet (TCVN 8338:2010) and meat (TCVN 7046:2009). Co-culturing bacteriocin and cellulose producing microorganisms would be investigated for further research on anti-microbial food packing.

\section{REFERENCES}

1. Azeredo, H.M.C., Barud, H., Farinas, C.S., Vasconcellos, V.M., Claro, A.M.(2019), “Bacterial cellulose as a raw material for food and food packaging applications", Frontiers in Sustainable Food Systems, 3(7).

2. Cotter, P.D., Hill, C., Ross, R.P. (2005), "Bacteriocins: developing innate immunity for food", Nature Reviews Microbiology, 3(10), 777-88.

3. Da Silva Sabo, S., Vitolo, M., Gonzalez, J.M.D., Oliveira, R.P.S. (2014), "Overview of Lac tobacillus plantarum as a promising bacteriocin producer among lactic acid bacteria", Food Research International, 64, 527-536.

4. Dos Santos, C.A., dos Santos, G.R., Soeiro, V.S., dos Santos, J.R., Rebelo, M.d.A., Chaud, M.V., Gerenutti, M., Grotto, D., Pandit, R., Rai, M., Jozala, A.F.(2018), "Bacterial nanocel lulose membranes combined with nisin: a strategy to prevent microbial growth", Cellulose, 25(11), 6681-6689.

5. De Vuyst, L., and Leroy, F. (2007),"Bacteriocins from lactic acid bacteria: production, purifi cation, and food applications", Journal of Molecular Microbiology and Biotechnology, 13(4), 194-199.

6. Kormin, S., Rusul G., Radu, S., Ling, F.H. (2001), "Bacteriocin-producing lactic acid bacteria isolated from traditional fermented food", The Malaysian journal of medical sciences: MJMS, 8(1), 63-68.

7. Joint annual health review (JAHR) (2015), Ministry of Health of Vietnam, Hanoi.

8. Nguyen T.H., Tran T.T.A. (2008), "Immobilizing Lactococcus lactic cell and applicating bacterial cellulose membrane treated with bacteriocin to preserving pork", Science \& Technology Development, 11(9), 777-88.

9. Ogunbanwo, S.T., Sanni, A.I., and Onilude A.A (2003), "Characterization of bacteriocin produced by Lactobacillus plantarum F1 and Lactobacillus brevis OG1", African Journal of Biotechnology, 2(8), 219-227.

10. Onda, T., et al. (2002), "Widespread distribution of the bacteriocin-producing lactic acid cocci in Miso-pasteproducts", Journalof applied microbiology, 92(4), 695-705.

11. Tagg, J.R., and McGiven A. R. (1971), “Assay system for bacteriocins", Applied microbiol ogy, 21(5), 943.

12. Todorov, S.D., Dicks, L.M.T. (2005), "Lactobacillus plantarum isolated from molasses produces bacteriocins active against Gram-negative bacteria". Enzyme and Microbial Technology, 36(2-3) 318-326.

13. Vignolo, Graciela M., et al. (1995), "Influence of growth conditions on the production of lactocin 705, a bacteriocin produced by Lactobacillus casei CRL705", Journal of Applied Bacteriology, 78(1) 5-10. 
Tóm tắt

\section{MÀNG PHÂN HỬY SINH HỌC KHÁNG KHUẨN BẢO QUẢN THỰC PHẨM SỐNG}

\section{Nguyễn Thị Huỳnh Nhu, Nguyễn Quỳnh Nhu, Nguyễn Thị Yến Nhi, Nguyễn Hoàng Ngọc Phương}

Khoa Sinh học \& Công nghệ Sinh học, Trương Đại học Khoa học Tụ nhiên, ĐHQG-HCM

Nguồn thịt cá tươi sống bày bán ngoài chợ thường phơi nhiễm với các tác nhân gây bệnh trong thời gian dài do các hộ tiểu thương không đầu tư thiết bị bảo quản thực phẩm chuyên dụng. Bên cạnh đó, bao nhựa dùng một lần đựng thực phẩm tươi sống trở thành gánh nặng đối với công tác xử lý rác thải và góp phần làm tình hình ô nhiễm trắng ngày càng trầm trọng. Bacteriocin là một chất kháng khuẩn tự nhiên, do vi khuẩn sinh lactic acid tổng hợp, từ lâu đã được ứng dụng trong bảo quản thực phẩm. Trong nghiên cứu này, chúng tôi thử nghiệm tạo màng cellulose vi sinh (bacterial cellulose, $\mathrm{BC}$ ) hấp phụ với bacteriocin để tạo màng bọc thực phẩm có khả năng phân hủy sinh học và hạn chế nhiễm khuẩn thực phẩm.

Màng bacterial cellulose được thu nhận từ quá trình lên men bề mặt của Acetobacterxylinum nuôi trong môi trường chứa $50 \%$ nước dừa và $15 \%$ sucrose sau 3 ngày đạt độ dày $2 \mathrm{~mm}$ và độ ẩm $94 \%$. LAB phân lập từ bắp cải chua, kim chi và sữa chua được kiểm tra bằng kính hiển vi, các phản ứng sinh hóa và định danh bằng khối phổ. Lactobacillus plantarum, dòng phân lập cho hoạt tính kháng khuẩn mạnh nhất trong 9 dòng đã phân lập, được nuôi cấy để thu bacteriocin thô đạt $100 \mathrm{AU} / \mathrm{ml}$ theo phương pháp khuếch tán trên giếng thạch tạo vòng kháng khuẩn to rõ so với đối chứng trên 3 chủng chỉ thị Escherichia coli, Staphylococusaureus và Bacillus subtilis. Màng $\mathrm{BC}$ hấp phụ bacteriocin nồng độ $100 \mathrm{AU} / \mathrm{mL}$ trong 45 phút có thể bảo quản thực phẩm tươi sống như cá trong 24 giờ (TCVN 8338:2010) và thịt trong 18 giờ (TCVN 7046:2009) ở nhiệt độ thường.

Tù khóa: Bacteriocin, màng cellulose, bảo quản thục phẩm, phân hủy sinh học. 